\title{
Frequency of early postoperative adverse events (AEs) in adult patients undergoing elective neurosurgical intervention at tertiary care center in Pakistan
}

\author{
Mansoor Chandio', \\ Faraz Shafiq ${ }^{2}$, Syed Ather Enam ${ }^{3}$
}

\begin{abstract}
Objective: The postoperative period is critical in neurosurgical patients, where the incidence of postoperative AEs is significantly high. Most of events occurs during recovery phase and has got relation to anaesthetic management. The objective of study was to determine frequency of early AEs in elective neurosurgical patients.
\end{abstract}

Methods: This cross sectional study was conducted at our tertiary care center. The duration of study was one year, from August 2017-July 2018. The data was collected using predesigned proforma. The assessment was done on arrival in recovery room (T1) and then at forty five minutes (T2), twenty-fourth hour (T3) and forty-eighth hour (T4) postoperatively.

Results: Total ninety-five patients were included. Overall, five hundred and forty AEs were recorded at T1, T2, T3 and T4. Anaesthesia related events like pain, postoperative sore throat, hoarseness, shivering and hypothermia were the commonest (73\%). There was a gradual decline in incidence of these events over period of 48 hours. There was no effect of age, sex, BMI and blood loss on incidence of AEs.

Conclusions: Postoperative pain, PONV and shivering were frequently reported AEs. We did not identify the impact of age, sex, BMI, comorbid or type of surgery in terms of having these events.

KEYWORDS: Neurosurgical procedures, Post-operative, Pakistan.

doi: https://doi.org/10.12669/pjms.37.4.3501

How to cite this:

Chandio M, Shafiq F, Enam SA. Frequency of early postoperative adverse events (AEs) in adult patients undergoing elective neurosurgical intervention at tertiary care center in Pakistan. Pak J Med Sci. 2021;37(4):939-944. doi: https://doi.org/10.12669/pjms.37.4.3501

This is an Open Access article distributed under the terms of the Creative Commons Attribution License (http://creativecommons.org/licenses/by/3.0), which permits unrestricted use, distribution, and reproduction in any medium, provided the original work is properly cited.

1. Dr. Mansoor Chandio, FCPS.

The Department of Anaesthesiology,

2. Dr. Faraz Shafiq FCPS.

The Department of Anaesthesiology,

3. Dr. Syed Ather Enam, MD, FRCS, PhD

The Department of Neurosurgery,

1-3: The Aga Khan University,

Stadium Road,

Karachi, Pakistan.

Correspondence:

Dr. Mansoor Chandio

Consultant Anesthetist,

The Aga Khan University,

Stadium Road,

Karachi, Pakistan.

Email: dr.mansoorhassan@gmail.com

* Received for Publication:

August 17, 2020

* Revision Received:

* Revision Accepted:

\section{INTRODUCTION}

The postoperative period is critical in neurosurgical patients, demands individualized and procedural specific care. The incidence of postoperative adverse events (AEs) in this patient population is higher as compared to other surgical procedures. ${ }^{1}$ The reported incidence is up to $24 \% .^{2}$ Most of the time these events occurs during immediate recovery phase. This relates not only to stress related to surgery, but anaesthetic intervention also plays major role in predicting short and long-term outcomes. ${ }^{3}$ However, association of AEs definitely worsens the outcome of neurosurgical patients and has direct impact on overall mortality. ${ }^{4}$ The early 
detection, rapid intervention and standardized care is key to successful management. ${ }^{5}$ This is not possible without having local data. In low middleincome countries (LMICs) where the resources are limited, the situation must be different. It is very difficult to determine the exact burden of these complications. The objective of this study was to determine the frequency of early AEs in elective neurosurgical patients at our tertiary care center.

\section{METHODS}

This cross sectional study was conducted at post anaesthesia care unit and neurosurgical ward, at Aga Khan University Hospital Karachi. The duration of study was one year, from August 2017 - July 2018. The study protocol was approved from Ethical review committee of Aga University Hospital on $16^{\text {th }}$ January 2017 bearing letter no 4409-Ane-ERC-16, and informed consent was taken from patients fulfilling inclusion criteria. Nonprobability consecutive sampling technique was used. All adult patients with age ranges between 18 to 70 years, undergoing elective neurosurgical procedures were included. The procedures were divided into either Cranial or Spinal surgery. Cranial surgeries included were elective tumor craniotomies. While procedures like laminectomy, discectomy, pedicle screw fixation and excision of spinal tumours were included as spinal surgical procedures. Patients already planned for postoperative intensive care unit (ICU) stay, had intraoperative major catastrophic event like cardiac arrest, myocardial infarction, major hypoxia and massive bleeding were excluded from study. Patients required unplanned ICU admission were also excluded from study protocol. The enrollment of patient into the study was started after hospital admission. The intraoperative anaesthetic care, monitoring, induction technique and maintenance of anaesthesia were at the discretion of primary anesthetist. After extubation, patients were shifted to PACU. An independent data collector was responsible for retrieving the data from patient progress notes and recording it on predesigned data collection form. All patients were reviewed immediately at the time of arrival in PACU (T1). The relevant clinical data was recorded from anaesthesia monitoring form. This included details about demographic data, anaesthetic care and surgical procedure. The patient was then reassessed at 45 minutes (T2) for any complications occurred at PACU. The recovery room chart was also reviewed. In ward, all patients were followed up at $24^{\text {th }}$ hour
(T3) and then; the final assessment was done at $48^{\text {th }}$ hour (T4) postoperatively. The file notes and daily progress sheets were reviewed for any AEs that occurred during ward stay. However, in case of any ambiguity in documentation, relevant teams were contacted to overcome any bias. For all time intervals that is; T1, T2, T3 and T4, legends were marked for any events, even if it already been marked for previous time interval. Recorded AEs were categorized as:

Anaesthesia Related: Airway trauma, postoperative pain, postoperative nausea/vomiting (PONV), postoperative sore throat (POST), shivering, and hypothermia.

Surgery Related: Position related injuries, seizures, reoperation, Cerebrospinal fluid (CSF) leak, drop in GCS, new onset of neurological deficit.

Cardiovascular: Hypertension (HTN), hypotension, arrhythmias, myocardial infarction (MI).

Pulmonary: Desaturation, re-intubation, hypoventilation, pulmonary edema, acute respiratory distress syndrome (ARDS), respiratory failure, pneumonia and pulmonary embolism (PE). Metabolic: Diabetes Insipidis (DI).

Following definitions were used to mark and log the AEs.

1. Postoperative pain/post craniotomy pain: Procedural pain, the assessment of which was done using Numeric Pain Rating scale (NPRS) of $1-10$. $^{6}$

2. PONV: As assessed on the PONV impact scale. ${ }^{7}$

3. Airway trauma: Any trauma to Lips, teeth or tongue associated with intubation, it also includes hoarsness of voice/laryngeal edema

4. POST: Postoperative pain in throat related in intubation, with no preoperative history of such pain before the operation. It was assessed using verbal rating scale of Yes or No.

5. Hypothermia: Core body temperature less than 36 degree Celsius.

6. Shivering: Involuntary movement associated with hypothermia. It was assessed using verbal rating scale of Yes or No.

7. CSF leak: Evident CSF leakage from nose or in the posterior pharynx as complained by patient and confirmed by surgical team.

8. Position related injuries: Pressure sores, burns injuries related to positioning during surgery.

9. Seizures: Any seizure activity occurred during postoperative period. It was using verbal rating scale of Yes or No.

10. Reoperation: Re-exploration required with in a period of 48 hours after procedure. 
11. New onset of Neurological deficit: Any cranial nerve, sensory or motor deficit.

12. Acute Drop in GCS: Sudden drop in GCS level for base line.

13. DI: Absolute or relative deficiency of Antidiuretic hormone postoperatively. This needs to be associated with increased urine volume and rise in serum sodium level. The diagnosis was made on the basis of clinical notes.

14. Pulmonary embolism: Symptomatic thromboembolic phenomena after surgery diagnosed after relevant investigations.

15. Hypertension: Systolic BP higher than 140 $\mathrm{mmHg}$ and diastolic higher than $90 \mathrm{mmHg}$.

16. Hypotension: Systolic BP less than $90 \mathrm{mmHg}$ and diastolic less than $60 \mathrm{mmHg}$.

17. Arrhythmias: Abnormal electrical rhythm of heart.

18. MI: Rise in serum Troponin levels with or without EKG evidence. This needs to be diagnosed by cardiology and documented accordingly.

19. Desaturation: Persistent drop in oxygen saturation $\left(\mathrm{SPO}_{2}\right)$ less than $92 \%$ requiring supplemental oxygen.

20. Need for re-intubation: The patient needs securing of airway because of neurological status i.e. GCS $<8 / 15$ or any other cause.

21. Hypoventilation: Respiratory rate of 8 breaths per minute.

22. Pulmonary edema: Abnormal accumulation of fluid in the extravascular compartments of the lung because of cardiac reasons.

23. Respiratory Failure: The term 'respiratory failure' is used when pulmonary gas exchange fails to maintain normal arterial oxygen and carbon dioxide levels.

24. ARDS: According to Berlins definition as follows.

Within 1 week of a known clinical

Timing insult or new/worsening respiratory symptoms.

Chest Bilateral opacities not fully explained ing (CT by effusions. Lobar/lung collapse or

Scan) nodules.

Respiratory failure not fully explained Ori- by cardiac failure or fluid overload.

gin of Needs objective assessment (e.g. echooedema cardiography) to exclude hydrostatic oedema if no risk factor present.

$\begin{array}{ll}\text { Oxy- } & \text { Mild }-26.6 \mathrm{kPa}<\mathrm{PaO} 2 / \mathrm{FIO} 2 \mathrm{PaO} 2 / \\ \text { gena- } & \mathrm{FIO} 2 \leq 39.9 \mathrm{kPa} \text { with PEEP or } \mathrm{CPAP} \geq \\ \text { tion } & 5 \mathrm{~cm} \mathrm{H}_{2} \mathrm{O} \\ & \text { Moderate }-13.3 \mathrm{kPa}<\mathrm{PaO} 2 / \mathrm{FI}- \\ & \mathrm{O} 2 \mathrm{PaO} 2 / \mathrm{FIO} 2 \leq 26.6 \mathrm{kPa} \text { with PEEP or } \\ & \mathrm{CPAP} \geq 5 \mathrm{~cm} \mathrm{H}_{2} 0 . \\ & \text { Severe }-\mathrm{PaO} 2 / \mathrm{FIO} 2 \mathrm{PaO} 2 / \mathrm{FIO} 2 \leq \\ & 13.3 \mathrm{kPa} \text { with } \mathrm{PEEP} \geq 5 \mathrm{~cm} \mathrm{H} \mathrm{H}_{2} \mathrm{O}\end{array}$

25. Bronchospasm: Characterized by a critical limitation of expiratory flow.

Statistical Analysis: Data was analyzed using statistical packages for social science version 19 (SPSS Inc., Chicago, IL). Frequency and percentage was computed for qualitative observations like gender, comorbid conditions like diabetes mellitus (DM), hypertension (HTN), ischemic heart disease (IHD), Asthma, and perioperative complications. Mean and standard deviation was estimated for age, height, weight, BMI, duration of surgical procedure and estimated blood loss. The frequency and percentages were also calculated for AEs at T1, T2, T3 and T4. Stratification analysis was done to evaluate the relationship between age, gender, BMI, comorbid, type of procedure and blood loos with the frequency of AEs. Chisquare test was used to observe difference. $\mathrm{P} \leq 0.05$ was considered as significant. The sample size was calculated on the basis of previous reported incidence of Post operative complications in neurosurgical patients; that is $54.5,{ }^{8}$ with $10 \%$ margin of error and $95 \%$ confidence interval.

\section{RESULTS}

Demographic characteristics of patients are shown in Table-I. The preoperative health status was measured using American Society of Anesthesiologists (ASA) status. $14.74 \%$ of the participants had ASA status I, $62.11 \%$ had ASA status II and $23.16 \%$ had ASA status III respectively. HTN was the common comorbid condition (32.6\%) followed by DM (11.6\%), IHD (2.1\%), and Asthma (2.1\%). Amongst surgical procedures 42 patients $(44.2 \%)$ had spinal, and 53(56\%) had cranial surgeries. The mean duration of surgical procedure was $240.39 \pm 118.08$ minutes. This was associated with mean blood loss of $486.68 \pm 454.88 \mathrm{ml}$ amongst our study participants. 14 patients were identified to have any intraoperative events (Table-II). Overall, 540 AEs were recorded at mentioned point intervals (T1, T2, T3 and T4). Amongst those, anaesthesia related events were at the top of the 
Table-I: Demographic characteristics of study patients.

\begin{tabular}{ll}
\hline Variables & Point estimation \\
\hline Age (years) & $43.04 \pm 14.29$ \\
BMI $\left(\mathrm{Kg} / \mathrm{m}^{2}\right)$ & $26.64 \pm 6.28$ \\
Duration of surgical & $240.39 \pm 118.08$ \\
procedure (minutes) & \\
Blood Loss (ml) & $486.68 \pm 454.88$ \\
Male & $(65.26 \%)$ \\
Female & $(34.74 \%)$ \\
ASA Status & \\
I & $(14.74 \%)$ \\
II & $(62.11 \%)$ \\
III & $(23.16 \%)$ \\
Comorbid Conditions & \\
Hypertension & $(32.6 \%)$ \\
Diabetic Mellitus & $(11.6 \%)$ \\
IHD & $(2.1 \%)$ \\
Asthma & $(2.1 \%)$ \\
Others & $(3.2 \%)$ \\
\hline
\end{tabular}

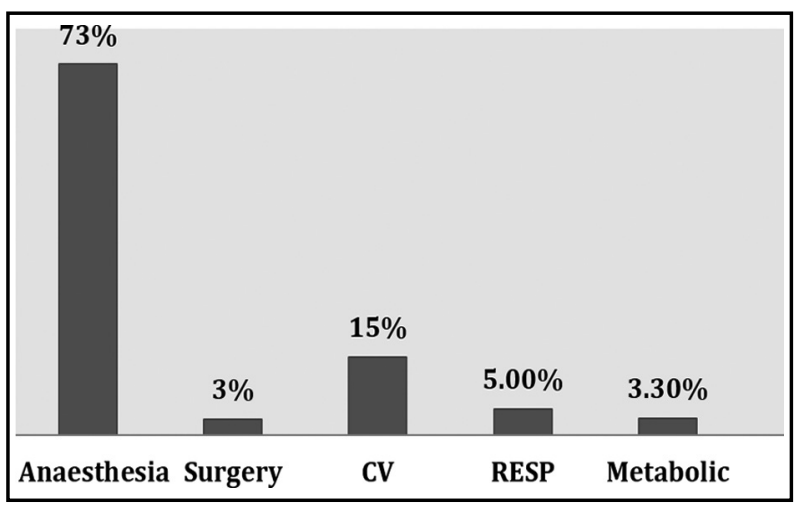

Fig.1: Overall frequency of AEs in neurosurgical patients.

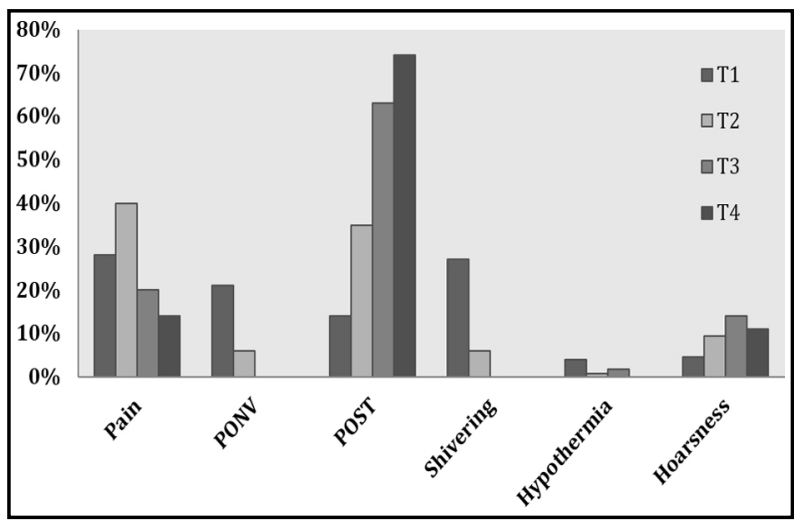

Fig.2: Comparison of anaesthesia related AEs at various time intervals.
Table-II: Perioperative information about study patients.

\begin{tabular}{ll}
\hline Variables & Percentage $\%$ \\
\hline Surgical Procedure & \\
Spinal & $44.2 \%$ \\
Cranial & $55.8 \%$ \\
Surgical Position & \\
Prone & $36.8 \%$ \\
Supine & $57.9 \%$ \\
Park Bench & $2.1 \%$ \\
Lateral & $3.2 \%$ \\
Invasive Monitoring & \\
Arterial Line & $66.3 \%$ \\
Central Venous Line & $23.2 \%$ \\
Any Intraopertive Event ( $\mathrm{n}=14)$ & $14.7 \%$ \\
Blood Loss (6) & $42 \%$ \\
Hemodynamic variation (6) & $42 \%$ \\
Difficult Intubation (1) & $7 \%$ \\
Different Bag mask ventilation (1) & $7 \%$ \\
\hline
\end{tabular}

list $(73 \%)$. This was followed by cardiovascular, respiratory, surgery and metabolic related events (Fig.1). There was a gradual decline in incidence of these events over a period of 48 hours, having peak at T1, while $\mathrm{T} 4$ was the time where the incidence was minimum (Table-III). The reported anaesthesia related events were pain, POST, hoarseness, shivering and hypothermia. At arrival in recovery room (T1), complain of having pain was at the top $(25 \%)$, followed by postoperative shivering $(22 \%)$. At $12^{\text {th }}$ hour (T2), pain and POST (30\% and 25\%) were the most frequent complications. While, POST was the main problem at $24^{\text {th }}(\mathrm{T} 3)$ and $48^{\text {th }}(\mathrm{T} 4)$ hour postoperatively (51\% and 60\%). (Fig.2). The severity of pain at various times interval is shown in Fig.3. Most patients had mild to moderate pain as measured on VAS.

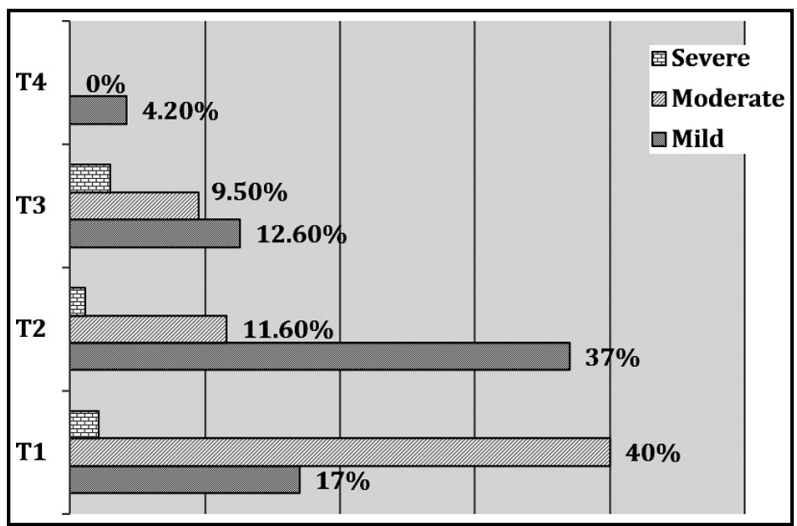

Fig.3: Severity of Pain at various time interval. 
Postoperative adverse events in elective neurosurgical intervention

Table-III: Frequencies of AEs at various time intervals.

\begin{tabular}{lccccc}
\hline Total Events $(n=540)$ & Overall (n) & T1 \% & T2\% & T3\% & T4\% \\
\hline Anaesthesia related & 397 & $49 \%$ & $29 \%$ & $15 \%$ & $7 \%$ \\
Surgery related & 16 & $37 \%$ & $31 \%$ & $19 \%$ & $13 \%$ \\
Cardiovascular & 83 & $55.4 \%$ & $34 \%$ & $6 \%$ & $5 \%$ \\
Respiratory & 26 & $85 \%$ & $14 \%$ & $4 \%$ & $0 \%$ \\
Metabolic & 18 & $33 \%$ & $33 \%$ & $33 \%$ & $0 \%$ \\
\hline
\end{tabular}

Stratification analysis found no difference of age, sex, BMI and blood loss on incidence of AEs. Similarly, we didn't identify any significant difference of these events in comparison to spinal versus cranial surgeries. However, metabolic events were more common in spinal surgeries $(\mathrm{P}=$ $0.073)$. Secondly, the pain was more in surgeries related to spine $(\mathrm{P}=0.040)$.

\section{DISCUSSION}

The postoperative period in our study showed significant association with AEs. The anaesthesia related events were at the top of the list. This corresponds to the results of various studies, which showed immedaite postoperative period as the high risk for such events. ${ }^{9}$ This requires vigilant monitoring, frequent assessments and standardized care of these patients. In contarst to various reports, we did not indentifed the effect of ASA status, age and comorbid conditions on reported complications. ${ }^{10}$ This must be related to the fact that most of the events study were directly related to anaesthesia and can affect any one irrespetive of base line health status. Similarly, we did not identify any difference in relation to cranial or spinal surgeries. However, metabolic complications like DI and SIADH were more common in patients having cranial surgeries. Frequency of anaesthesia related AEs need to be focused here. At the time of arrival in PACU pain, shivering and PONV (28\%:27\%:21\%) were most frequently reported.

At T2, pain and POST (40\%:35\%) were the most common. The same pattern was followed at intervals of $\mathrm{T} 3$ and $\mathrm{T} 4$, where the incidence of POST was $63 \%$ and $74 \%$ respectively. Most of our patients had mild to moderate pain as assessed using NPRS. However, we are not following any set protocol for postoperative pain management which could be the reason of this reporting. Studies have shown better pain management with the implementation of standarized protocol using patient controlled analgesia (PCIA). ${ }^{11}$

The overall incidence of PONV is bit low. This must be because of double antiemetic prophylaxis with Dexamethasone and Ondansteron, which we usually follow for our neurosurgical patients. The efficacy of regime is proven through various studies $^{12}$ and the results here is validating the same. HTN and desaturation were the most frequent cardiorespiratory events recorded in this study. Most of these events were occur at T1 and T2. However, none of these patients required intuabtion or unplanned ICU admission. Though studies have shown residual neuromuscular blockage as the cause of respiratory compliations. The reported incidence is between 2 to 35 . Postoperative HTN in our study could be directly related to pain or postopertaive shivering. We think that targeting both would resolve the issue related to HTN.

In contrast to results of various studies reporting hypotension during recovery phase, ${ }^{13}$ we didn't observe this in our study population. Only seven patients had hypotensive episode at T1. The events related to surgery were very low. This could be directly related to the facts that patients here were elective, and well optimized. Only two patients reported dropped in GCS in PACU. Similarly only one patient had seizure episode postoperatively. The very low incidence of seizures in our cohort is reinforcing the efficacy of prophylactice use of Levetiracetam. ${ }^{14}$ Its routine for us to prescribe seizure prophylaxis after surgery. There are certain limitations associated with this study. First, the data is from elective neurosurgical patient only, that may not be the true reflection. Moreover, we didn't followed the patients beyond 48 hours which might have missed the long term outcomes. Secondly, the data is from single centre which may not reflect the staus nationwide. 


\section{CONCLUSIONS}

In our elective neurosurgical patients, postoperative pain, nausea, vomiting and shivering were the frequently reported AEs. All of anaesthesia related events were peak at immediate recovery phase (T1 and T2) and got setteled with the passage of time (T3 and T4). We did not identify the impact of age, sex, BMI and comorbid conditions in terms of having these complications. Similarly, there was no significant difference in comparision to occurrence of these events in cranial versus spinal procedures.

\section{Acknowledgements:}

1: Dr. Hamid Iqil Naqvi: For his input during write up.

2: Amir Raza: Statistical Analysis.

3: Nausheen Abbas: For data compilation and filter.

1,2,3: Department of Anaesthesiology, The Aga Khan University, Stadium Road, Karachi, Pakistan.

Grant Support \& Financial Disclosures: None.

\section{REFERENCES}

1. Noordzij PG, Poldermans D, Schouten O, Bax JJ, Schreiner FA, Boersma E. Postoperative mortality in The Netherlands: A population-based analysis of surgeryspecific risk in adults. Anesthesiology. 2010;112(5):11051115. doi: 10.1097/ALN.0b013e3181d5f95c

2. Rolston JD, Han SJ, Lau CY, Berger MS, Parsa AT. Frequency and predictors of complications in neurological surgery: National trends from 2006 to 2011. J Neurosurg. 2014;120(3):736-745. doi: 10.3171/2013.10.JNS122419

3. Todd MM. Outcomes after neuroanesthesia and neurosurgery: what makes a difference. Anesthesiol Clin. 2012;30(2):399-408. doi: 10.1016/j.anclin.2012.06.001

4. De la Garza-Ramos R, Kerezoudis P, Tamargo RJ, Brem $\mathrm{H}$, Huang J, Bydon M. Surgical complications following malignant brain tumor surgery: An analysis of 2002-2011 data. Clin Neurol Neurosurg. 2016;140:6-10. doi: 10.1016/j. clineuro.2015.11.005

5. Hall AJ, Toner NS, Bhatt PM. The introduction of a Neurosurgical Postoperative Checklist improved quality of care and patient safety. Br J Neurosurg. 2019;33(5):495499. doi: 10.1080/02688697.2019.1606894

6. Alghadir AH, Anwer S, Iqbal A, Iqbal ZA. Test-retest reliability, validity, and minimum detectable change of visual analog, numerical rating, and verbal rating scales for measurement of osteoarthritic knee pain. J Pain Res. 2018;11:851-856. doi: 10.2147/JPR.S158847
7. Myles PS, Wengritzky R. Simplified postoperative nausea and vomiting impact scale for audit and post-discharge review. Br J Anaesth. 2012;108(3):423-429. doi: 10.1093/ bja/aer505

8. Manninen PH, Raman SK, Boyle K, el-Beheiry H. Early postoperative complications following neurosurgical procedures. Can J Anaesth. 1999;46(1):7-14. doi: 10.1007/ BF03012507

9. Ayrian E, Kaye AD, Varner CL, Guerra C, Vadivelu $\mathrm{N}$, Urman RD, et al. Effects of Anesthetic Management on Early Postoperative Recovery, Hemodynamics and Pain After Supratentorial Craniotomy. J Clin Med Res. 2015;7(10):731-741. doi: 10.14740/jocmr2256w.

10. Westerkamp AC, de Geus AF, Molenbuur B, et al. Comparing peri-operative complications of paediatric and adult anaesthesia: A retrospective cohort study of 81267 cases. Eur J Anaesthesiol. 2018;35(4):280-288. doi: 10.1097/ EJA.0000000000000769

11. Vacas S, Van de Wiele B. Designing a pain management protocol for craniotomy: A narrative review and consideration of promising practices. Surg Neurol Int. 2017;8:291. doi: 10.4103/sni.sni_301_17

12. Thongrong C, Chullabodhi P, Kasemsiri P, Kitkhuandee A, Plailaharn N, Sabangban L, et al. Effects of Intraoperative Dexamethasone and Ondansetron on Postoperative Nausea and Vomiting in Microvascular Decompression Surgery: A Randomized Controlled Study. Anesthesiol Res Pract. 2018;2018:6297362. doi: 10.1155/2018/6297362

13. Munting KE, van Zaane B, Schouten AN, Wolfswinkel $\mathrm{LV}$, de Graaff JC. Reporting critical incidents in a tertiary hospital: a historical cohort study of 110,310 procedures. Can J Anaesth. 2015;62:1248-1258. doi: 10.1007/s12630015-0492-y

14. Gokhale S, Khan SA, Agrawal A, Friedman AH, McDonagh DL. Levetiracetam seizure prophylaxis in craniotomy patients at high risk for postoperative seizures. Asian J Neurosurg. 2013;8(4):169-173. doi: 10.4103/17935482.125658

\section{Authors' Contribution:}

MC conceived, designed and did statistical analysis. Also responsible and accountable for the accuracy and integrity of work.

MC, FS did data collection and manuscript writing and editing of manuscript.

SA did review and manuscript writing and design of study. 